\title{
Phase-Based User-Steered Image Segmentation
}

\author{
Lauren O’Donnell ${ }^{1}$, Carl-Fredrik Westin ${ }^{1,2}$, W. Eric L. Grimson ${ }^{1}$, \\ Juan Ruiz-Alzola ${ }^{3}$, Martha E. Shenton ${ }^{2}$, and Ron Kikinis ${ }^{2}$ \\ 1 MIT AI Laboratory, Cambridge MA 02139, USA \\ odonnell, welg@ai.mit.edu \\ 2 Brigham and Women's Hospital, Harvard Medical School, Boston MA, USA \\ westin, kikinis@bwh.harvard.edu \\ 3 Dept. of Signals and Communications, University of Las Palmas de Gran Canaria, \\ Spain \\ jruiz@dsc.ulpgc.es
}

\begin{abstract}
This paper presents a user-steered segmentation algorithm based on the livewire paradigm. Livewire is an image-feature driven method that finds the optimal path between user-selected image locations, thus reducing the need to manually define the complete boundary. We introduce an image feature based on local phase, which describes local edge symmetry independent of absolute gray value. Because phase is amplitude invariant, the measurements are robust with respect to smooth variations, such as bias field inhomogeneities present in all MR images. In order to enable validation of our segmentation method, we have created a system that continuously records user interaction and automatically generates a database containing the number of user interactions, such as mouse events, and time stamps from various editing modules. We have conducted validation trials of the system and obtained expert opinions regarding its functionality.
\end{abstract}

\section{Introduction}

Medical image segmentation is the process of assigning labels to voxels in order to indicate tissue type or anatomical structure. This labeled data has a variety of applications, for example the quantification of anatomical volume and shape, or the construction of detailed three-dimensional models of the anatomy of interest.

Existing segmentation methods range from manual, to semi-automatic, to fully automatic. Automatic algorithms include intensity-based methods, such as EM segmentation [14] and level-sets [4, as well as knowledge-based methods such as segmentation by warping to an anatomical atlas. Semi-automatic algorithms, which require user interaction during the process, include snakes [11] and livewire [1.7. Current practice in many cases, however, is computer-enhanced manual segmentation. This involves hand-tracing around the structures of interest and perhaps employing morphological or thresholding operations to the data.

Although manual segmentation methods allow the highest degree of user control and enable decisions to be made on the basis of extensive anatomical knowledge, they require excessive amounts of user interaction time and may 
introduce high levels of variability. On the other hand, fully automatic algorithms may not produce the correct outcome every time, hence there exists a need for hybrid methods.

\section{Existing Software Platform}

The phase-based livewire segmentation method has been integrated into the 3D Slicer, the Surgical Planning Lab's platform for image visualization, image segmentation, and surgical guidance [9]. The Slicer is the laboratory's program of choice for manual segmentation, as well as being modular and extensible, and consequently it is the logical place to incorporate new algorithms for our user base. At any time during an average day, one would expect to find ten people doing segmentations using the 3D Slicer in the laboratory, which shows that any improvement on manual methods will be of great utility. Our system has been added into the Slicer as a new module, Phasewire, in the Image Editor.

\section{The Livewire Algorithm}

The motivation behind the livewire algorithm is to provide the user with full control over the segmentation while having the computer do most of the detail work [1]. In this way, user interaction complements the ability of the computer to find boundaries of structures in an image.

Initially when using this method, the user clicks to indicate a starting point on the desired contour, and then as the mouse is moved it pulls a "live wire" behind it along the contour. When the user clicks again, the old wire freezes on the contour, and a new live one starts from the clicked point. This intuitive method is driven only by image information, with little geometric constraint, so extracting knowledge from the image is of primary importance.

The livewire method poses this problem as a search for shortest paths on a weighted graph. Thus the livewire algorithm has two main parts, first the conversion of image information into a weighted graph, and then the calculation of shortest paths in the graph 612].

In the first part of the livewire algorithm, weights are calculated for each edge in the weighted graph, creating the image forces that attract the livewire. To produce the weights, various image features are computed in a neighborhood around each graph edge, transformed to give low edge costs to more desirable feature values, and then combined in some user-adjustable fashion. The general purpose of the combination is edge localization, but individual features are generally chosen for their contributions in three main areas, which we will call "edge detection," "directionality," and "training." Features such as the gradient and the Laplacian zero-crossing [13] have been used for edge detection; these features are the main attractors for the livewire. Second, directionality, or the direction the path should take locally, can be influenced by gradients computed with oriented kernels [7], or the "gradient direction" feature [13]. Third, training is the 
process by which the user indicates a preference for a certain type of boundary, and the image features are transformed accordingly. Image gradients and intensity values [7, as well as gradient magnitudes [13, are useful in training.

In the second part of the livewire algorithm, Dijkstra's algorithm [5] is used to find all shortest paths extending outward from the starting point in the weighted graph. The livewire is defined as the shortest path that connects two user-selected points (the last clicked point and the current mouse location). This second step is done interactively so the user may view and judge potential paths, and control the segmentation as finely as desired. The implementation for this paper is similar to that in 6], where the shortest paths are computed for only as much of the image as necessary, making for very quick user interaction.

\section{Phase-Based Livewire}

\subsection{Background on Local Phase}

The local phase as discussed in this paper is a multidimensional generalization of the concept of instantaneous phase, which is formally defined as the argument of the analytic function

$$
f_{A}=f-i f_{H i}
$$

where $f_{H i}$ denotes the Hilbert transform of $f[23$.

The instantaneous phase of a simple one-dimensional signal is shown in Figure 1 This simple signal can be thought of as the intensity along a row of pixels in an image. Note that in this "image" there are two edges, or regions where the signal shape is locally odd, and the phase curve reacts to each, giving phase values of $\pi / 2$ and $-\pi / 2$.
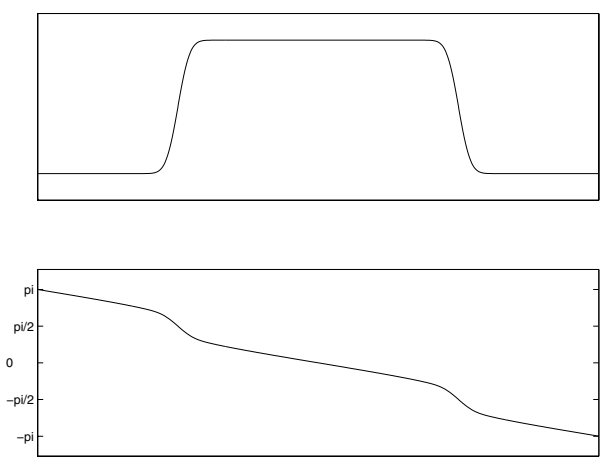

Fig. 1. A simple signal (top), and its instantaneous phase (bottom).

The output from a local phase filter, i.e. a Gabor filter, is an analytic function that can be represented as an even real part and an odd imaginary part, or an 
amplitude and an argument. Consequently, this type of filter can be used to estimate both the amplitude (energy) and the local phase of a signal. As local phase is invariant to signal energy, edges can be detected from small or large signal variations. This is advantageous for segmentation along weak as well as strong edges. Another advantage is that local phase is in general a smoothly varying function of the image, and provides subpixel information about edge position.

\subsection{Implementation}

To estimate the phase we are using quadrature filters which have a radial frequency function that is Gaussian on a logarithmic scale:

$$
\nu(\rho)=\mathrm{e}^{-\frac{4}{\log 2} B^{-2} \log ^{2}\left(\frac{\rho}{\rho_{i}}\right)}
$$

where $\rho_{i}$ is the center frequency and $B$ is the $6 \mathrm{~dB}$ sensitivity bandwidth in octaves. In contrast to Gabor filters, these filters have zero response for negative frequencies, ensuring that the odd and even parts constitute a Hilbert transform pair and making the filters ideal for estimation of local phase [10]. We multiply the filters with a $\cos ^{2}$ radial function window in the frequency domain to reduce ringing artifacts arising from the discontinuity at $\pi$ when using filters with high center frequencies and/or large bandwidth.

The local phase is our primary feature, serving to localize edges in the image: the livewire essentially follows along low-cost curves in the phase image. The phase feature is scaled to provide bounded input to the shortest-paths search. Figure 2 shows a phase image derived from the original image shown in Figure 4

Since the argument of the phase is not sensitive to the amount of energy in the signal, it is useful to also quantify the certainty of the phase estimate. In image regions where the phase magnitude is large, the energy of the signal is high, which implies that the the phase estimate in that region has high reliability and thus high certainty.

We define the certainty using the magnitude from the quadrature filters, mapped through a gating function. By inverting this certainty measure, it can be used as a second feature in the livewire cost function. The weighted combination of the phase and certainty images produces an output image that describes the edge costs in the weighted graph, as in Figure 2

\section{Applications}

In this section, we describe the behavior of our phase-based segmentation technique through a series of experiments with medical images.

In the first set of experiments, we show the stability of phase information across different scales [8]. The input to the quadrature filters was blurred using a Gaussian kernel of variance 4 pixels. Figure 3 demonstrates the robustness 


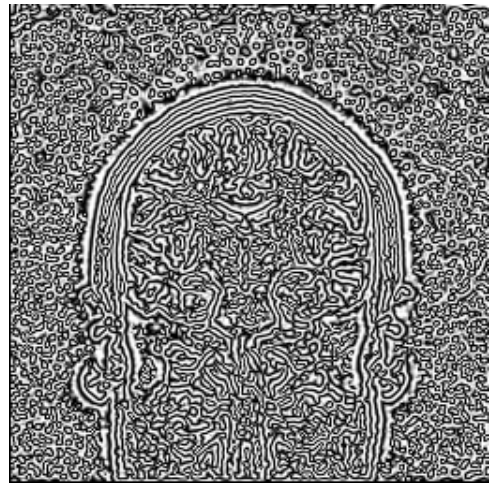

Local Phase Image

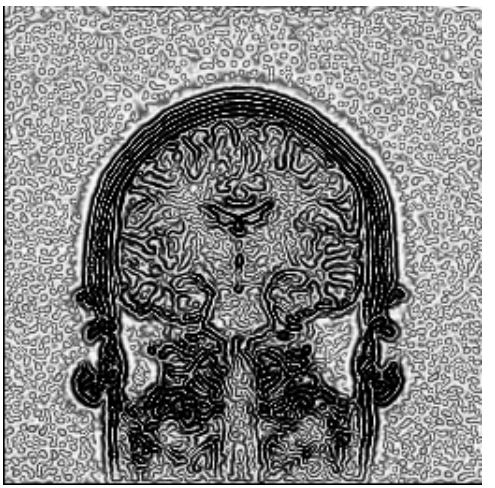

Combined Image

Fig. 2. Local phase images. The left-hand image shows the local phase corresponding to the grayscale image in Figure 4. This is the primary feature employed in our phase-based livewire method. The right-hand image displays the weighted combination of the phase and certainty features, which emphasizes boundaries in the image. Darker pixels correspond to lower edge costs on the weighted graph.

of phase information to changes in scale, as the two segmentations are quite similar. The leftmost image is the original grayscale and its segmentation, while the center image shows the result of Gaussian blurring. The rightmost image displays the segmentation curve from the blurred image over the original image for comparison.

The second experiments contrast the new livewire feature, phase, with our original intensity-based livewire implementation. Phase is more intuitive to use, produces smoother output, and is generally more user-friendly than the intensitybased method as illustrated in Figure 4. In contrast to the intensity-based method, no training step is required to bias the phase-based method towards the desired edge type, as the phase information can be used to follow weak or strong edges.

\section{Discussion and Validation Framework}

We have presented a user-steered segmentation algorithm which is based on the livewire paradigm. Our preliminary results using local phase as the main driving force are promising and support the concept that phase is a fairly stable feature in scale space 8]. The method is intuitive to use, and requires no training despite having fewer input image features than other livewire implementations.

In order to enable validation of phase-based livewire, we have created a system that continuously records user interaction and can generate a database containing the number of user interactions and time stamps from various editing modules. A selection of the items which are recorded is listed in Table 1 An 


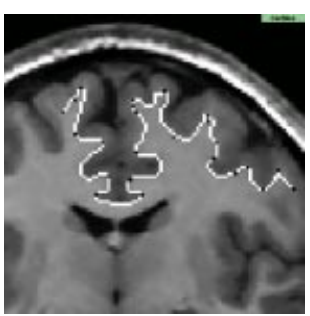

Original Image
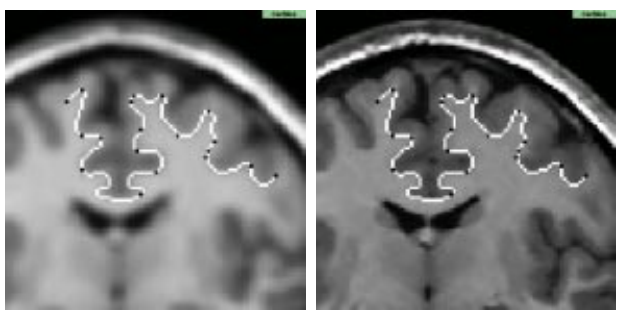

Gaussian Blurring Overlay of Segmentation

Fig. 3. Illustration of insensitivity to changes in scale: initial image, result of Gaussian blurring, and overlay of second segmentation (done on the blurred image) on initial image. Note the similarity between the segmentation contours, despite blurring the image with a Gaussian kernel of four pixel variance.

ongoing study using this system will enable evaluation of the performance and utility of different image features for guiding the livewire.

Analysis of the logged information has shown that great variability exists in segmentation times, partially due to users' segmentation style, learning curve, and type of image data, and also due to factors we cannot measure, such as time spent using other programs with the Slicer Editor window open. A complete comparison of the phasewire and manual segmentations systems, consequently, is difficult, and perhaps the most reliable indication of the system performance can be found in the opinions of doctors who have used it.

Table 2 demonstrates the utility of the segmentation method using information from the logging system, and Table 3 gives doctors' opinions regarding ease of use and quality of output segmentations. For example, Figure 5 shows a liver segmentation done with phasewire, in which the doctor remarked that the three-dimensional model was more anatomically accurate than the one produced with the manual method, where both segmentations were performed in approximately the same amount of time. This is likely due to the smoothness of the contour obtained with phasewire, in comparison with the contour obtained with the manual method.

\begin{tabular}{ll}
\hline Item Logged & \multicolumn{1}{c}{ Details } \\
\hline mouse clicks & recorded per slice, per label value, per volume, and per editor module \\
elapsed time & recorded per slice, per label value, per volume, and per editor module \\
description & includes volumes viewed and location of scene description file \\
username & will allow investigation of learning curve \\
\hline
\end{tabular}

Table 1. Selected items logged by the 3D Slicer for validation and comparison purposes. 

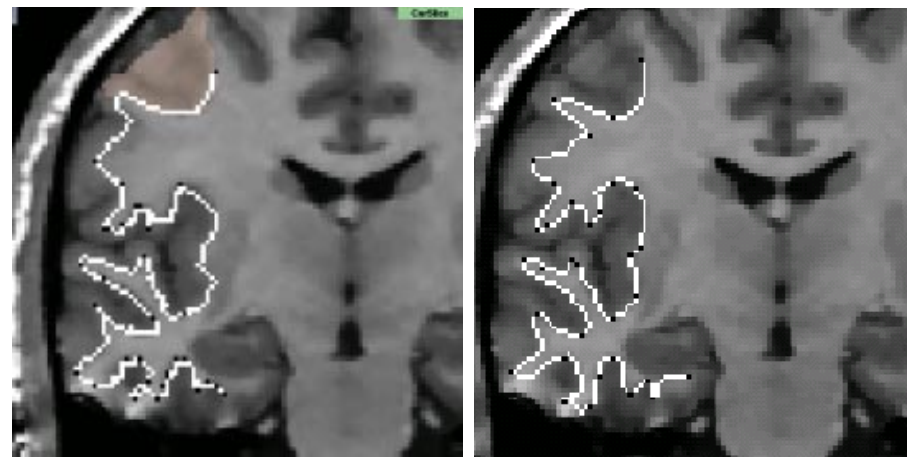

Fig. 4. Comparison of phase-based and intensity-based implementations of livewire. On the left is our original implementation of livewire, which uses imageintensity based features. It does a poorer job of segmenting gray and white matter. Training was performed on the region in the upper left of the image. The segmentation was more difficult to perform than the phase-based segmentation because more coercing was necessary to force the livewire to take an acceptable path. The right image shows segmentation of the same boundary using the phase-based livewire. Contrary to the intensity-based method, no training was necessary before beginning to segment. The conclusion is that the phase-based implementation is more intuitive to use than the intensity-based version, since the phase image gives continuous contours for the livewire to follow. Rather than being distracted by unwanted pixels locally, the phase livewire is generally only distracted by other paths, and a small movement of the mouse can set it back on the desired path. It can be quite fluid to use, with clicks mainly necessary when the curve bends in order to keep the wire following the correct contour.

\section{Acknowledgements}

The work was funded in part by CIMIT and NIH P41-RR13218 (RK,CFW), by a National Science Foundation Graduate Research Fellowship (LJO), by NIH R01 RR11747 (RK), by Department of Veterans Affairs Merit Awards, NIH grants K02 MH 01110 and R01 MH 50747 (MS), and by European Commission and Spanish Gov. joint research grant 1FD97-0881-C02-01 (JR). Thanks to the Radiation Oncology, Medical Physics, Neurosurgery, Urology, and Gastroenterology Departments of Hospital Doctor Negrin in Las Palmas de Gran Canaria, Spain, for their participation in evaluation of the system. Also thanks to Aleksandra Ciszewski for her valuable feedback regarding the Slicer system.

\section{References}

1. W. A. Barrett and E. N. Mortensen. Interactive live-wire boundary extraction. Medical Image Analysis, 1(4):331-341, 1997. 


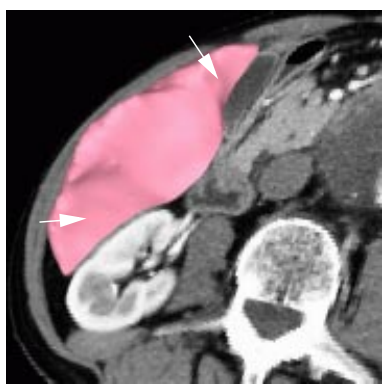

Fig. 5. A surface model of the liver, created from a phasewire segmentation, shows well-defined surfaces between the liver and the kidney and gallbladder.

\begin{tabular}{llllll}
\hline study & method & total clicks & clicks/slice & time/slice (sec) & volume (mL) \\
\hline \multirow{2}{*}{ CT brain tumor manual } & 234 & 26.0 & 39.3 & 68.5 \\
& phasewire & 97 & 10.8 & 28.7 & 67.3 \\
& phasewire & 109 & 12.1 & 25.5 & 69.2 \\
\hline \multirow{2}{*}{ CT bladder } & & & & & \\
& manual & 1488 & 28.1 & 31.7 & 715.6 \\
& phasewire & 359 & 6.8 & 21.5 & 710.8 \\
\hline
\end{tabular}

Table 2. Example segmentations performed with Phasewire. Clicks and time per slice are averages over the dataset.

2. Boualem Boashash. Estimating and interpreting the instantaneous frequency of a signal - part 1: Fundamentals. Proceedings of the IEEE, 80(4), 1992.

3. Boualem Boashash. Estimating and interpreting the instantaneous frequency of a signal - part 2: Algorithms and applications. Proceedings of the IEEE, 80(4), 1992.

4. V. Caselles, R. Kimmel, G. Sapiro, C. Sbert. Minimal Surfaces: A Three Dimensional Segmentation Approach. Technion EE Pub 973, 1995.

5. E. W. Dijkstra A Note On Two Problems in Connexion With Graphs Numerische Mathematik, 269-271, 1959.

6. A. X. Falcao, J. K. Udupa, and F. K. Miyazawa. An Ultra-Fast User-Steered Image Segmentation Paradigm: Live Wire on the Fly. IEEE Transactions on Medical Imaging, 19(1):55-61, 2000.

7. A. X. Falcao, J. K. Udupa, S. Samarasekera, and S. Sharma. User-Steered Image Segmentation Paradigms: Live Wire and Live Lane. Graphical Models and Image Processing, 60:233-260, 1998.

8. D. J. Fleet and A. D. Jepson. Stability of Phase Information IEEE Trans. PAMI, 15(12):1253-1268, 1993.

9. D. T. Gering, A. Nabavi, R. Kikinis, W. E. L. Grimson, N. Hata, P. Everett, and F. Jolesz and W. Wells. An Integrated Visualization System for Surgical Planning and Guidance Using Image Fusion and Interventional Imaging. Medical Image Computing and Computer-Assisted Intervention - MICCAI'99, 809-819, 1999. 


\begin{tabular}{llll}
\hline study & method & ease of use & segmentation quality \\
\hline \multirow{2}{*}{ CT brain tumor } & manual & 3 & 3 \\
& phasewire & 4 & 4 \\
\hline \multirow{2}{*}{ CT bladder } & manual & 2.7 & 3 \\
& phasewire & 4 & 4 \\
\hline
\end{tabular}

Table 3. Doctors' comparison of Phasewire and Manual segmentation methods on the datasets from Table 2. The scale is from 1 to 5 , with 5 being the highest.

10. H. Knutsson and C.-F. Westin and G. H. Granlund. Local Multiscale Frequency and Bandwidth Estimation Proceedings of IEEE International Conference on Image Processing, 36-40, 1994.

11. T. McInerney, D. Terzopoulos. Deformable Models in Medical Image Analysis: A Survey. Medical Image Analysis, 1(2):91-108, 1996.

12. E. N. Mortensen and W. A. Barrett. Interactive Segmentation with Intelligent Scissors. Graphical Models and Image Processing, 60(5):349-384, 1998.

13. E. N. Mortensen and W. A. Barrett. Intelligent Scissors for Image Composition. Computer Graphics (SIGGRAPH '95), 191-198, 1995.

14. W. M. Wells, R. Kikinis, W.E.L. Grimson, F. Jolesz. Adaptive segmentation of MRI data. IEEE Transactions on Medical Imaging, 15:429-442, 1996. 